\title{
Padronizando a Exposição à Radiação durante o Cateterismo Cardíaco em Crianças com Cardiopatia Congênita: Dados de um Registro Multicêntrico Brasileiro
}

\author{
Standardizing Radiation Exposure during Cardiac Catheterization in Children with Congenital Heart Disease: \\ Data from a Multicenter Brazilian Registry
}

\author{
João Luiz Manica, ${ }^{1 \oplus}$ Vanessa Oliveira Duarte, ${ }^{1}$ Marcelo Ribeiro, ${ }^{2}$ Adam Hartley, ${ }^{3}$ Ricardo Petraco, ${ }^{3}$ Carlos Pedra, ${ }^{2}$ \\ Raul Rossi $i^{1}$ \\ Instituto de Cardiologia / Fundação Universitária de Cardiologia (IC/FUC), ${ }^{1}$ Porto Alegre, RS - Brasil \\ Hospital do Coração, ${ }^{2}$ São Paulo, SP - Brasil \\ National Heart and Lung Institute, Imperial College London, Hammersmith Hospital, ${ }^{3}$ Londres - Reino Unido
}

\section{Resumo}

Fundamento: Nos últimos anos, o recente aumento no número de procedimentos intervencionistas tem resultado em crescente preocupação em relação à exposição radiológica por pacientes e equipe médica. A avaliação da exposição dos níveis de radiação em crianças é difícil devido à grande variabilidade no peso corporal. Portanto, os valores de referência de radiação não estão bem definidos para essa população.

Objetivos: Avaliar e validar a razão do produto dose-área (DAP) em relação ao peso corporal como uma medida de referência de radiação em cateterismos cardíacos em crianças.

Métodos: Estudo multicêntrico observacional com dados do Registro Brasileiro de Cateterismo Cardíaco em Cardiopatias Congênitas (CHAIN) de março de 2013 a junho de 2014. Os critérios de inclusão foram: pacientes $<18$ anos submetidos a procedimentos hemodinâmicos para cardiopatia congênita, com DAP devidamente registrado. Foram considerados diferenças estatísticas significativas os valores de $\mathrm{p}<0,05$.

Resultados: Este estudo avaliou 429 pacientes com idade e peso medianos de $50(10,103)$ meses e $15(7,28) \mathrm{kg}$, respectivamente. O DAP mediano foi de $742,2(288,8,1.791,5) \mu \mathrm{Gy} . \mathrm{m} 2$. Houve uma boa correlação entre o DAP e o produto peso/tempo de fluoroscopia ( $r s=0,66)$. Não foi observada diferença estatisticamente significativa na relação DAP/peso entre procedimentos terapêuticos e diagnósticos. Houve ampla variação da relação DAP/peso entre os procedimentos terapêuticos $(p<0.001)$.

Conclusões: A proporção DAP/peso é a medida mais simples e aplicável para avaliar a exposição radiológica em uma população pediátrica. Apesar da escassa literatura disponível, as doses obtidas no presente estudo foram semelhantes àquelas encontradas anteriormente. Estudos de validação e comparação são importantes na avaliação do impacto de estratégias para redução da exposição radiológica nessa população. (Arq Bras Cardiol. 2020; 115(6):1154-1161)

Palavras-chave: Diagnóstico por Imagem/métodos, Vias de Exposição de Radiação, Cardiopatias Congênitas, Cateterismo Cardíaco/métodos, Criança

\begin{abstract}
Background: In recent years the increasing number of interventional procedures has resulted in growing concerns regarding radiation exposure for patients and staff. The evaluation of radiation exposure in children is difficult due to the great variability in body weight. Therefore, reference levels of radiation are not well defined for this population.
\end{abstract}

Objectives: To study and validate the ratio of dose-area product (DAP) to patient weight as a reference measurement of radiation for hemodynamic congenital heart disease procedures in children.

Methods: This observational multicenter study uses data obtained from a Brazilian registry of cardiac catheterization for congenital heart disease from March 2013 to June 2014. Inclusion criteria were all patients aged <18 years old undergoing hemodynamic procedures for congenital heart disease, with recorded DAP doses. P-value $<0.05$ was considered as statistically significant.

Correspondência: João Luiz Manica •

Instituto de Cardiologia / Fundação Universitária de Cardiologia (IC/FUC) - Avenida Princesa Isabel, 395. CEP 90620-000, Porto Alegre, RS - Brasil E-mail: joca.pesquisa@gmail.com

Artigo recebido em 07/01/2019, revisado em 07/11/2019, aceito em 27/12/2019

DOI: https://doi.org/10.36660/abc.20190012 
Results: This study evaluated 429 patients with median age and weight of $50(10,103)$ months and 15 (7, 28$) \mathrm{kg}$, respectively. Median DAP was $742.2(288.8,1,791.5) \mu$ Gy.m2. There was a good correlation between DAP and weight-fluoroscopic time product(rs=0.66). No statistically significant difference was observed in DAP/weight ratio between therapeutic and diagnostic procedures. There was a wide variation in the DAP/ weight ratio among the therapeutic procedures $(p<0.001)$.

Conclusions: The DAP/weight ratio is the simplest and most applicable measurement to evaluate radiation exposure in a pediatric population. Although there is limited literature available, the doses obtained in the present study were similar to those previously found. Ongoing research is important to evaluate the impact of strategies to reduce radiation exposure in this population. (Arq Bras Cardiol. 2020; 115(6):1154-1161)

Keywords: Diagnostic,Imaging/methods; Radiation Exposure Pathways; Heart Defects, Congenital; Cardiac Catheterization/methods; Child

Full texts in English - http://www.arquivosonline.com.br

\section{Introdução}

Nos últimos 20 anos, o cateterismo cardíaco não só foi utilizado como exame diagnóstico de cardiopatias congênitas, mas também desempenhou um papel importante nos tratamentos paliativo e definitivo de mais de $50 \%$ dos pacientes com cardiopatias congênitas. ${ }^{1}$ Nesse período, a complexidade, a duração e o número de procedimentos percutâneos aumentaram, além de um consequente aumento da exposição dos pacientes à radiação ionizante. ${ }^{2-4}$

As crianças são altamente sensíveis à radiação ionizante, devido à maior proporção de células em divisão ativa e à grande fração da área corporal exposta. ${ }^{2}$ Assim, existe uma grande preocupação com os efeitos cumulativos, particularmente o alto risco de malignidade causado por danos cromossômicos a longo prazo, com relatos demonstrando que crianças são até dez vezes mais suscetíveis ao desenvolvimento de câncer por exposição à radiação do que adultos. ${ }^{5,6}$ Além disso, a dose de radiação efetiva é maior para crianças, resultando em uma dose de radiação mais alta para os órgãos vizinhos quando uma área de interesse está sendo avaliada.

Há poucos estudos sobre doses de radiação emitidas durante intervenções em crianças com cardiopatia congênita. 3,7 Para obter redução da dose de radiação, é essencial estabelecer doses de referência que permitam comparações entre procedimentos. ${ }^{4}$ No entanto, é difícil avaliar a exposição à radiação em uma população pediátrica devido às diferenças nas complexidades dos procedimentos, idade e peso dos pacientes, bem como nos tipos de equipamentos utilizados. ${ }^{8}$ Além disso, o cálculo da dose efetiva estimada de radiação é complexo. Atualmente, a dose total de radiação (kerma no ar total) e o produto dose-área total (dose-area product - DAP), que é a melhor forma de estimar os efeitos estocásticos (efeitos de radiação a longo prazo e risco de malignidade) e efeitos cumulativos da exposição, são utilizados como indicadores de uma dose cumulativa de radiação na pele.

Recentemente, Chida et al. ${ }^{2}$ e Kobayashi et al. ${ }^{8}$ observaram correlação entre DAP e peso como referência de dose de radiação em crianças. Eles concluíram que a dose de radiação tende a variar proporcionalmente ao tamanho do paciente. Nesse contexto, o presente estudo tem como objetivo avaliar a razão DAP/peso como referência de exposição a radiação em procedimentos de cateterismo cardíaco pediátrico realizados no Brasil.

\section{Materiais e Métodos}

\section{Desenho e População do Estudo}

Trata-se de um estudo observacional transversal em que pacientes com $<18$ anos de idade e participantes do registro de Intervenção e Angiografia de Cardiopatias Congênitas (Congenital Heart Disease Intervention and Angiography CHAIN), um registro brasileiro de cateterismo cardíaco para cardiopatia congênita, foram avaliados após um diagnóstico ou procedimento de intervenção entre 5 de março de 2013 e 30 de junho de 2014 .

O registro CHAIN é um estudo prospectivo nacional multicêntrico, coordenado pelo Instituto de Ensino e Pesquisa do Hospital do Coração, em conjunto com o Ministério da Saúde e a Sociedade Brasileira de Hemodinâmica e Cardiologia Intervencionista. O principal objetivo foi reunir dados prospectivos e criar um registro nacional de cateterismo de pacientes com cardiopatias congênitas, além de propor uma análise abrangente da situação atual e elaborar medidas efetivas de ação para a saúde pública no Brasil.

Pacientes submetidos a procedimentos eletrofisiológicos ou aqueles em que o acesso vascular foi obtido por meio de procedimentos híbridos foram excluídos do estudo. Pacientes submetidos a mais de um cateterismo em datas diferentes foram considerados pacientes distintos em cada procedimento e incluídos nas estatísticas gerais, bem como no grupo de cada procedimento específico. Os pacientes submetidos a mais de uma intervenção utilizando o mesmo procedimento foram classificados de acordo com o procedimento mais complexo.

\section{Variáveis Analisadas}

Características demográficas dos pacientes, como idade, sexo, peso, superfície corporal, tipo de cardiopatia e lesões residuais, foram obtidas através do registro CHAIN, além de dados referentes ao procedimento hemodinâmico realizado, incluindo tempo fluoroscópico e dose de exposição à radiação. O DAP, que representa a dose de radiação medida no ar em relação à distância do tubo de raios-X multiplicada pela área do feixe de raios- $X$ a essa distância, foi expresso em $\mu \mathrm{Gy} . \mathrm{m}^{2}$. As medidas de radiação expressas em unidades de Gy.cm² cGy. $\mathrm{cm}^{2}$ e mGy. $\mathrm{cm}^{2}$ foram convertidas e registradas em $\mu \mathrm{Gy}$. $\mathrm{m}^{2}$. Além disso, a razão DAP/peso $\left(\mu \mathrm{G} y \cdot \mathrm{m}^{2} / \mathrm{kg}\right)$ foi analisada entre as categorias de cateterismo para possíveis comparações e padronização das doses de radiação. Procedimentos sem dados relacionados à dose de radiação, ou dose de radiação registrada em diferentes unidades, foram excluídos do estudo. 
Os procedimentos de cateterismo terapêutico foram divididos em 10 categorias. A exposição à radiação foi avaliada após a categorização dos pacientes em subgrupos de idade ( $<1$ ano; 1 a 4 anos; 5 a 9 anos; 10 a 14 anos; $\geq$ 15 anos) e peso (até $7 \mathrm{~kg}$; até $15 \mathrm{~kg}$; até $28 \mathrm{~kg}$; $>28 \mathrm{~kg}$ ). Os dados referentes a: DAP, razão DAP/peso, idade, peso, tempo fluoroscópico e produto peso-fluoroscópico não eram normalmente distribuídos e, portanto, eram descritos como medianas (intervalo interquartil).

\section{Análise Estatística}

Todos os dados foram analisados usando o SPSS (IBM, SPSS Statistics, Versão 22.0. Armonk, NY: IBM Corp).

O método Kolmogorov Smirnov foi o teste estatístico utilizado para verificar a normalidade dos dados. As variáveis contínuas não apresentaram distribuição normal após a aplicação do teste de Kolmogorov-Smirnov. As variáveis quantitativas não normalmente distribuídas são apresentadas como medianas (intervalo interquartil). As variáveis categóricas são apresentadas como frequências absolutas (n). As associações entre variáveis contínuas foram avaliadas pelo teste do coeficiente de correlação de Spearman $\left(r_{s}\right)$. A relação entre variáveis quantitativas contínuas não paramétricas e duas variáveis categóricas foi avaliada pelo teste $U$ de MannWhitney. A relação entre variável quantitativa contínua não paramétrica e mais de duas variáveis categóricas foi avaliada pelo teste de Kruskal-Wallis. O valor de $\mathrm{p}<0,05$ foi considerado estatisticamente significante.

\section{Resultados}

A análise incluiu um total de 1.311 pacientes com idade $<18$ anos de 16 centros participantes do estudo CHAIN. Entre esses, 206 pacientes não tinham registro de doses de radiação e foram excluídos. Dos 1.026 pacientes restantes com doses registradas de radiação, 597 foram excluídos porque suas doses não foram registradas como DAP. Isso resultou em um total de 429 pacientes participantes (56,4\% do sexo masculino) de seis centros. Após a aplicação desses critérios de exclusão, três dos seis centros contribuíram com 90\% dos dados dos pacientes.

Os dados demográficos e as características da população e grupos de procedimentos estão descritos na Tabela 1.
O DAP mediano na população estudada foi de 742,2 $(288,8,1.791,5) \mu \mathrm{Gy} . \mathrm{m}^{2}$. Os procedimentos intervencionistas apresentaram DAP mediano mais alto que os procedimentos de diagnóstico: 751 (315, 2.095) versus 715 (230, 1.535) $\mu \mathrm{Gy} . \mathrm{m}^{2}$, respectivamente. Não foram observadas diferenças na razão $\mathrm{DAP} /$ peso entre os procedimentos diagnóstico e terapêutico: $57(23,110)$ versus $57(30,139)$, respectivamente.

Verificou-se que o DAP apresenta uma boa correlação com o produto do tempo peso-fluoroscópico $\left(r_{s}=0,66\right)$, e esse padrão de correlação também foi observado quando os procedimentos diagnósticos e terapêuticos foram analisados separadamente $\left(r_{\mathrm{s}}=0,56\right.$ e $r_{\mathrm{s}}=0,72$, respectivamente) (Figuras 1 e 2). Pacientes categorizados em subgrupos de peso demonstraram doses mais altas de radiação (DAP) em procedimentos terapêuticos do que em diagnósticos $(p=0,001)$. Quando os pacientes foram categorizados em subgrupos etários, observou-se diferença significativa nas doses de radiação entre procedimentos diagnósticos e terapêuticos, mas apenas em pacientes com idade $>15$ anos ( $p=0,004 ;$ Tabela 2).

A Tabela 3 destaca os diferentes procedimentos, tempos fluoroscópicos e as proporções correspondentes de DAP/ peso. As maiores razões de DAP/peso foram observadas para implante valvar pulmonar percutâneo (Melody), fechamento de defeitos do septo ventricular (DSV) e angioplastia com balão ou stent na via de saída do ventrículo direito (VSVD) ou artéria pulmonar (PA), com médias de 273,8, 169,2 e 155,9 , respectivamente. Além disso, houve uma diferença significativa entre os subgrupos do procedimento de intervenção e as proporções DAP/peso ( $p<0,001)$.

\section{Discussão}

Nos últimos anos, a complexidade e o número de procedimentos transcateter aumentaram. ${ }^{4}$ Assim, métodos para proteger pacientes e funcionários da exposição cumulativa à radiação ionizante e seus potenciais efeitos são importantes e, portanto, o estabelecimento de dados de referência é crucial. ${ }^{8}$ Atualmente, as principais limitações para estabelecer valores de referência com procedimentos intervencionistas para cardiopatias congênitas são a falta de padronização das unidades de dosagem e medida ${ }^{9}$ e

Tabela 1 - Dados demográficos e características dos procedimentos

\begin{tabular}{|c|c|c|c|c|}
\hline & & Procedimentos diagnósticos & Procedimentos intervencionistas & $p$ \\
\hline Pacientes & 429 & 151 & 278 & \\
\hline Idade (meses) & $50,1(10,102,9)$ & $38,8(13,6,104,5)$ & $53(9,2,102,6)$ & 0,892 \\
\hline Peso (kg) & $15(7,2,28)$ & $12(7,2,27)$ & $16(7,1,29,5)$ & 0,466 \\
\hline Tempo de procedimento (min) & $40(27,5,57)$ & $35(25,50)$ & $45(30,60)$ & 0,000 \\
\hline Tempo fluoroscópico (min) & $9(5,15)$ & $8(4,13)$ & $9(5,7,16)$ & 0,003 \\
\hline Peso x tempo fluoroscópico (kg.min) & $114(54,5,250)$ & $90(45,224)$ & $128(60,277)$ & 0,006 \\
\hline DAP (uGy. m²) & $742(288,8,1.791,8)$ & $715,2(230,1.534,9)$ & $751,5(315,4,2.095,2)$ & 0,14 \\
\hline DAP/peso (uGy.m²/kg) & $57,2(28,124,9)$ & $57(23,3,110,5)$ & $57(30,5,139,5)$ & 0,137 \\
\hline
\end{tabular}

Os resultados são descritos em medianas e intervalo interquartil (percentil 25, 75). DAP: produto dose-área. Significância estatística quando $p \leq 0,05$. 


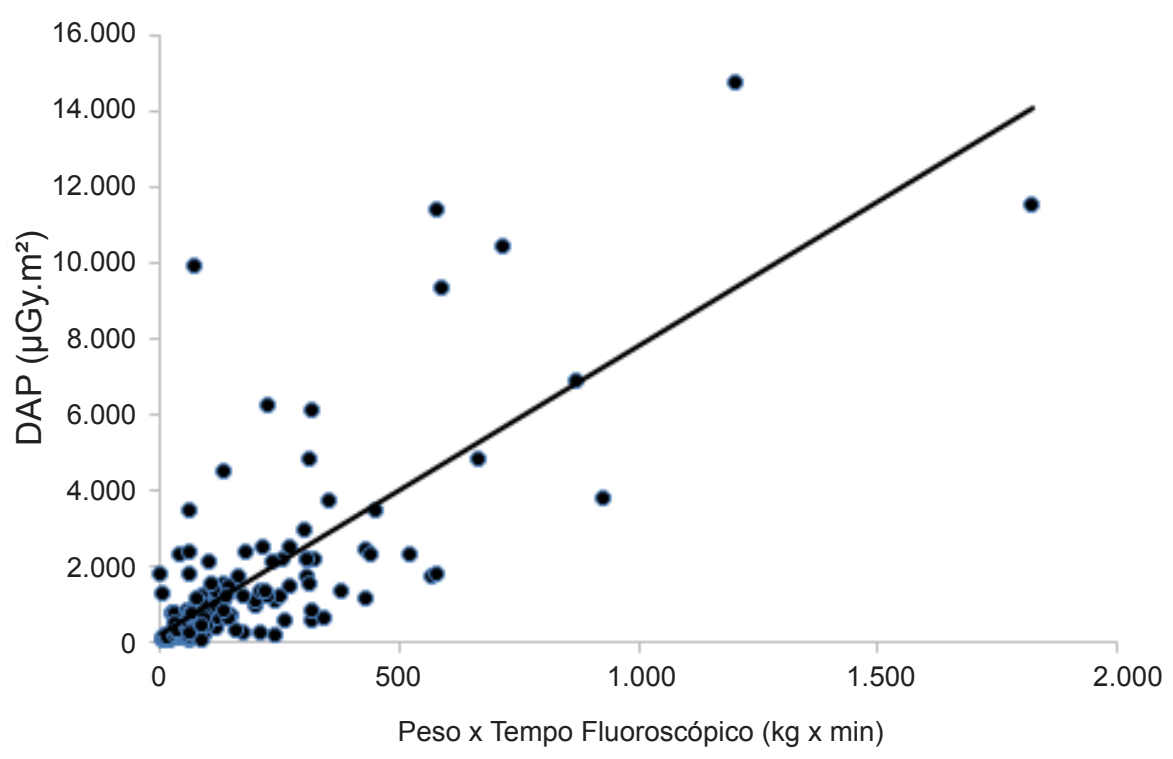

Figura 1 - Gráfico de dispersão mostra a relação entre produto dose-área (DAP) e peso-produto tempo fluoroscópico em pacientes pediátricos submetidos a cateterismo cardíaco diagnóstico $(r=0,75)$.

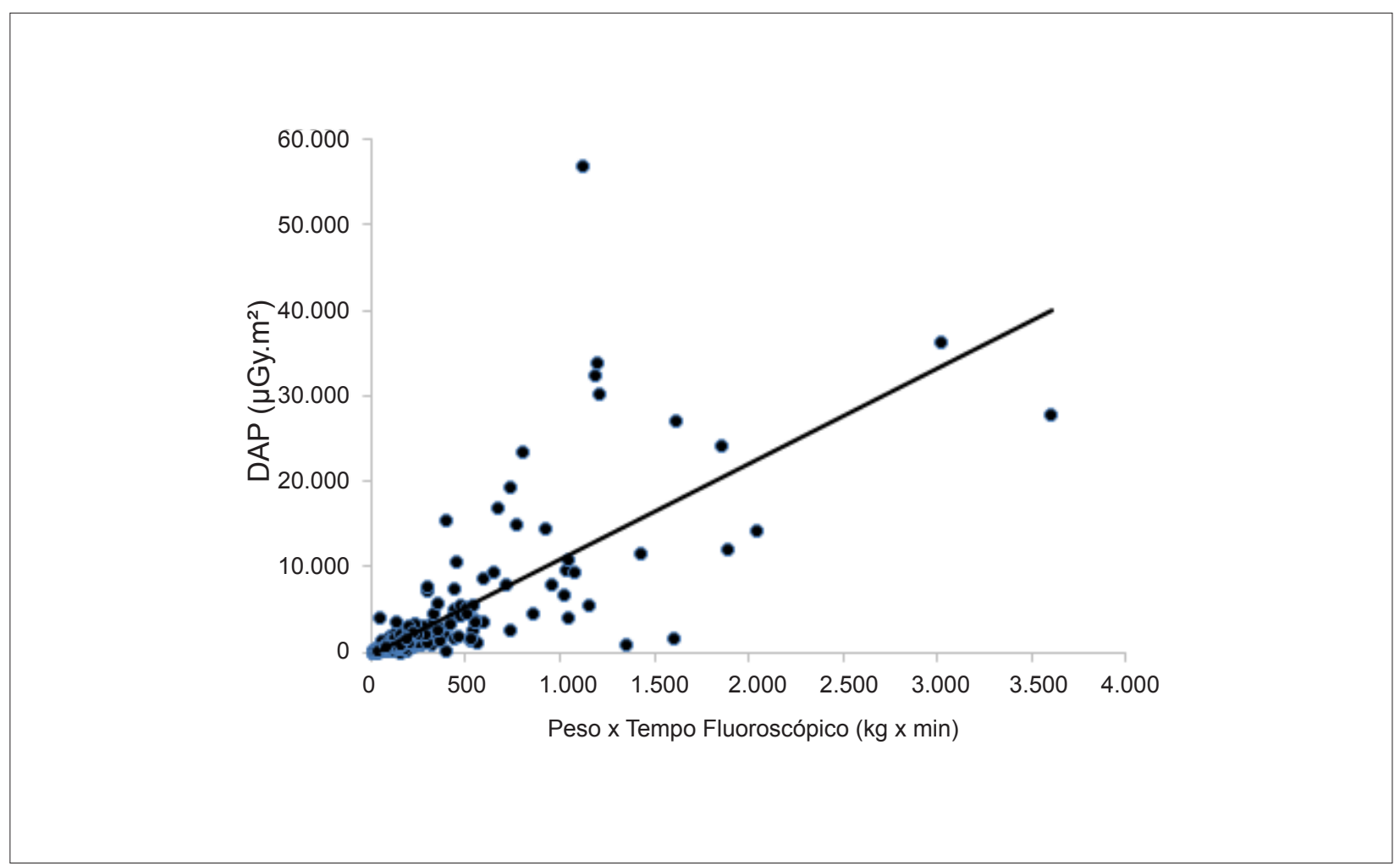

Figura 2 - 0 gráfico de dispersão mostra a relação entre o produto dose-área (DAP) e o produto tempo fluoroscópico-peso em pacientes pediátricos submetidos a cateterismo cardíaco terapêutico $(r=0,74)$. 


\section{Artigo Original}

Tabela 2 - Produto dose-área (DAP; uGy.m²) dos cateterismos diagnósticos e terapêuticos estratificados por faixas etárias

\begin{tabular}{|c|c|c|c|}
\hline \multicolumn{4}{|c|}{ Tipo de cateterismo } \\
\hline Faixa etária & Diagnóstico & Terapêutico & $\mathrm{p}$ \\
\hline$<1$ ano & $\begin{array}{c}n=36 \\
303,8(172 ; 754)\end{array}$ & $\begin{array}{c}n=78 \\
250,7(138,6 ; 570,7)\end{array}$ & 0,25 \\
\hline 1-4 anos & $\begin{array}{c}n=50 \\
524,8(194,3 ; 1.038,7)\end{array}$ & $\begin{array}{c}n=73 \\
602,7(409,5 ; 1.329,3)\end{array}$ & 0,06 \\
\hline 5-9 anos & $\begin{array}{c}n=75 \\
1.340(428,9 ; 2.175,9)\end{array}$ & $\begin{array}{c}n=71 \\
1.189,7(491,7 ; 2.125,4)\end{array}$ & 0,82 \\
\hline 10-14 anos & $\begin{array}{c}n=119 \\
1.739,6(773,7 ; 4.524,5)\end{array}$ & $\begin{array}{c}n=38 \\
2.765(1.385,3 ; 8.399,4)\end{array}$ & 0,08 \\
\hline$>15$ anos & $\begin{array}{c}n=11 \\
2.182,2(295,1 ; 3.735,7)\end{array}$ & $\begin{array}{c}n=18 \\
11.723,5(5.493,5 ; 28.357,2)\end{array}$ & 0,004 \\
\hline
\end{tabular}

Valores de DAP descritos em medianas e intervalos interquartis (percentil 25, 75). $n$ : número absoluto de pacientes. Significância estatística quando $p \leq 0,05$.

Tabela 3 - Tempo fluoroscópico e produto dose-área normalizado indexado ao peso corporal (DAP/peso; uGy.m²/kg) estratificados por tipos de procedimentos

\begin{tabular}{lcccc}
\hline & Pacientes & $\%$ & Tempo fluoroscópico & DAP/peso (uGy.m²/kg) \\
\hline Diagnóstico & 151 & 37,3 & $8(4 ; 13)$ & $57,2(23 ; 110,5)$ \\
\hline Valvoplastia pulmonar & 44 & 10,9 & $10(7 ; 15)$ & $51,8(35 ; 93)$ \\
\hline Valvoplastia aórtica & 20 & 4,9 & $9(7 ; 13)$ & $59,8(29,1 ; 125,9)$ \\
\hline Oclusão de PDA & 56 & 13,8 & $6(5 ; 9)$ & $41,9(27,6 ; 71,4)$ \\
\hline Fechamento do dispositivo DSA/FOP & 52 & 12,8 & $5(4 ; 7,7)$ & $25,5(13,5 ; 36,2)$ \\
\hline Fechamento do dispositivo DSV & 6 & 1,5 & $17(10 ; 44)$ & $155,9(71,4 ; 513,4)$ \\
\hline Angioplastia ou stent VSVD/AP & 35 & 8,6 & $11(6 ; 1) 24,5)$ \\
\hline Angioplastia aórtica / & 32 & 7,9 & $9(8,5 ; 15,5)$ & $98,2(42 ; 206,6)$ \\
Stent aórtico & 6 & 1,5 & $36(\mathrm{p} 25=34)$ & $77,2(58 ; 126,6)$ \\
\hline Stent PDA & 3 & 0,7 & $273,8(\mathrm{p} 25=41,9)$ \\
\hline Implante de válvula Melody & &
\end{tabular}

Tempo de fluoroscopia e valores de DAP descritos em medianas e intervalos interquartis (percentil 25, 75)

$n=$ número absoluto de pacientes. Significância estatística quando $p \leq 0,05$. PDA: ducto arterioso patente; DAS: defeitos do septo atrial; FOP: forame oval patente; DSV: defeito do septo ventricular; VSVD: via de saída do ventrículo direito; AP: artéria pulmonar.

a existência de uma ampla variedade de procedimentos e complexidades, variações de peso e idade, tipos de equipamentos e habilidades médicas. Todos esses fatores contribuem para uma grande heterogeneidade, o que dificulta comparações. ${ }^{4,8}$ O Food and Drug Administration e a Organização Mundial da Saúde recomendam o registro do DAP e o cálculo de doses efetivas para todos os pacientes submetidos a procedimentos que utilizam radiação. ${ }^{10} \mathrm{Com}$ base nessa proposta, avaliaram-se neste momento 429 pacientes com idade $<18$ anos e registrados no estudo CHAIN. Embora relativamente menor que o número de pacientes relatados em estudos anteriores, , , , ,8,11,12 os resultados da presente análise revelam o potencial do uso da razão DAP/ peso como referência para comparação.

A ausência de diferença estatística no DAP entre os procedimentos diagnósticos e terapêuticos no presente estudo pode ser explicada pelos recentes avanços nos procedimentos intervencionistas de baixa complexidade, como o fechamento percutâneo de defeitos do septo atrial (DSA), forame oval patente (PFO), e ducto arterioso patente (Patent ductus arteriosus - PDA), além da valvoplastia pulmonar, que utiliza doses de radiação relativamente baixas. Além disso, os procedimentos de diagnóstico geralmente envolvem pacientes com doenças cardíacas complexas sem um diagnóstico definido, exigindo alto tempo de fluoroscopia.

Durante a análise do cateterismo diagnóstico e terapêutico, observou-se que o DAP aumentou à medida que a idade aumentou. Quando os dois procedimentos foram comparados com os subgrupos etários, não foram observadas diferenças estatísticas, exceto no grupo com idade > 15 anos, em que a dose de radiação foi significativamente maior nos procedimentos terapêuticos, semelhante ao relatado por Ubeda et al. ${ }^{13}$ Isso provavelmente foi resultado do maior número de procedimentos complexos, como implante valvar percutâneo e angioplastia em pacientes mais velhos. 
Os principais procedimentos intervencionistas analisados no presente estudo tiveram medianas de dose comparáveis às relatadas em estudos recentes ${ }^{3-8,11,13,14}$ (Tabela 3), principalmente quando os valores foram comparados pela razão DAP/peso, que padroniza valores crescentes de DAP relacionado às diferenças de peso no mesmo procedimento. A variação da razão DAP/peso entre os diferentes tipos de cateterismo intervencionista foi estatisticamente significante, como demonstrado em outros estudos. , $, 8,11,12,14^{\text {As maiores }}$ doses de radiação foram observadas em implantes valvares pulmonares percutâneos (Melody), fechamento de DSV e angioplastias com balão ou stent em VSVD ou AP, como relatado anteriormente. ${ }^{8,11}$ As medianas da razão DAP/ peso em valvoplastias pulmonares, fechamento de DSV e angioplastias com balão ou stent de VSVD ou AP foram semelhantes às obtidas por Kobayashi e Borik et al. ${ }^{8,11}$

Em muitos procedimentos no presente estudo, as medianas do DAP foram inferiores às observadas em estudos anteriores. ${ }^{3,4,15}$ Glatz et al. ${ }^{15}$ avaliaram 2.265 pacientes em um estudo de centro único e obtiveram um DAP mediano significativamente maior que na maioria dos procedimentos estudados, mas o estudo incluiu adultos e pacientes com peso > $65 \mathrm{~kg}$ (máximo, $128 \mathrm{~kg}$ ). Por outro lado, o estudo CHAIN apresentou um peso mediano de $21 \mathrm{~kg}$. O único procedimento relatado por Glatz et al. com uma dose menor que a do presente estudo foi a aortoplastia com balão/stent (DAP de 484 versus 1,904 $\mu \mathrm{Gy} . \mathrm{m}^{2}$, respectivamente). Ghelani et al. publicaram um estudo realizado de 2009 a 2011 com 2.713 pacientes nos quais o DAP de alguns procedimentos intervencionistas foi avaliado. As medianas relatadas do DAP foram superiores às de outros estudos, incluindo o estudo CHAIN. Esses resultados também podem ser parcialmente justificados pela inclusão de pacientes com idade $>15$ anos e adultos, representando aproximadamente 20\% da população avaliada. No entanto, neste estudo, a relação $\mathrm{DAP} / \mathrm{Kg}$ não foi avaliada. Todos esses dados corroboram o conceito de que o uso da razão DAP/peso é uma medida racional para padronizar a avaliação da dose de radiação em uma população pediátrica heterogênea. De acordo com essa linha de pensamento, Cevallos et al publicaram recentemente novos parâmetros de referência para dosagem de radiação na população pediátrica. Diferentemente do estudo anterior do mesmo grupo ${ }^{4}$, eles avaliaram o DAP/kg estratificado por faixas etárias e tipos de procedimentos, o que permite a comparação com a literatura atual. ${ }^{12}$ Este estudo foi realizado após os esforços de melhoria da qualidade da radiação (MQ) nos diferentes centros envolvidos. Curiosamente, as doses médias encontradas por nosso grupo no presente foram muito semelhantes às relatadas por Cevallos et al. após um programa de MQ (Tabela 4).

A principal limitação do presente estudo foi a falta de dados de alguns centros participantes, provavelmente devido à ausência de padronização dos dados coletados. Como conseqüência, a amostra estudada foi menor e possivelmente menos heterogênea. Ao mesmo tempo, isso corrobora a hipótese de falta de padronização das medidas de exposição à radiação em populações pediátricas e demonstra que vários centros brasileiros ainda não relatam adequadamente a dose de radiação usada em seus procedimentos. Isso reforça a necessidade de conscientização das instituições em relação a um controle apropriado e a um programa de garantia de qualidade bem desenvolvido para segurança contra radiação. Além disso, em algumas análises o número de pacientes avaliados foi pequeno e portanto a análise estatística não foi possível, por como exemplo, no implante valvar pulmonar percutâneo. No entanto, as doses de radiação que esses pacientes receberam foram semelhantes às citadas na literatura.

\section{Conclusão}

A dose de radiação aumenta com a idade do paciente e a complexidade do procedimento. No presente estudo, as doses de radiação observadas foram semelhantes às de outros estudos relatados. As doses de radiação nesses procedimentos devem servir de referência para outras instituições para o controle apropriado da exposição à radiação de pacientes e funcionários.

A proporção DAP/peso parece ser a medida de radiação mais útil e aplicável para o estabelecimento de uma dose de referência para a população pediátrica, uma vez que permite a eliminação de categorias etárias e engloba o amplo espectro de tamanhos corporais. Dessa forma, novos estudos utilizando a razão DAP/peso são importantes para o desenvolvimento de doses de referência em procedimentos hemodinâmicos e para a avaliação de estratégias, visando reduzir a exposição à radiação de pacientes e funcionários.

\section{Contribuição dos Autores}

Concepção e desenho da pesquisa: Manica J, Ribeiro $M$, Pedra C, Rossi R; Obtenção de dados: Manica J, Duarte V, Ribeiro M, Pedra C, Rossi R; Análise e interpretação dos dados: Manica J, Duarte V; Análise estatística: Duarte V, Petraco R; Redação do manuscrito: Manica J, Duarte V, Hartley A, Petraco R; Revisão crítica do manuscrito quanto ao conteúdo intelectual importante: Petraco R, Pedra C, Rossi R.

\section{Potencial Conflito de Interesses}

Declaro não haver conflito de interesses pertinentes.

\section{Fontes de Financiamento}

O presente estudo não teve fontes de financiamento externas.

\section{Vinculação Acadêmica}

Não há vinculação deste estudo a programas de pósgraduação.

\section{Aprovação Ética e Consentimento Informado}

Este estudo foi aprovado pelo Comitê de Ética do Instituto de Cardiologia/Fundação Universitária de Cardiologia (IC/ FUC) sob o número de protocolo 2.919.655. Todos os procedimentos envolvidos nesse estudo estão de acordo com a Declaração de Helsinki de 1975, atualizada em 2013. O consentimento informado foi obtido de todos os participantes incluídos no estudo. 
Tabela 4 - Comparação de nossos dados estratificados por tipo de procedimentos, dados de radiação de procedimentos (CHAIN) com bancos de dados de doses de radiação publicados anteriormente

\begin{tabular}{|c|c|c|c|c|c|c|c|c|c|c|}
\hline \multirow{2}{*}{ Procedimentos } & \multicolumn{2}{|c|}{$\begin{array}{l}\text { Manica, } 2018 \\
\text { (CHAIN) }\end{array}$} & \multicolumn{2}{|c|}{ Cevallos, 2017 (C3PO) } & \multicolumn{2}{|c|}{ Borik, 2015} & \multicolumn{2}{|c|}{$\begin{array}{l}\text { Kobayashi, } 2014 \\
\text { (CCISC) }\end{array}$} & \multicolumn{2}{|c|}{ Onnasch, 2007} \\
\hline & $\mathrm{n}$ & a DAP/peso & $\mathrm{n}$ & ${ }^{\mathrm{b}} \mathrm{DAP} /$ peso & $\mathrm{n}$ & a DAP/peso & $\mathrm{n}$ & c DAP/peso & $\mathrm{n}$ & ${ }^{c}$ DAP/peso \\
\hline $\begin{array}{l}\text { Valvoplastia } \\
\text { pulmonar }\end{array}$ & 44 & $\begin{array}{c}51,8 \\
(34-92)\end{array}$ & 258 & $\begin{array}{c}53 \\
(104-335)\end{array}$ & 286 & $\begin{array}{c}28 \\
(1-345)\end{array}$ & 342 & $\begin{array}{c}56 \\
(152)\end{array}$ & - & - \\
\hline Valvoplastia aórtica & 20 & $\begin{array}{c}59,8 \\
(29-126)\end{array}$ & 136 & $\begin{array}{c}99 \\
(165-383)\end{array}$ & 138 & $\begin{array}{c}42 \\
(8-211)\end{array}$ & 138 & $\begin{array}{c}80 \\
(127)\end{array}$ & - & - \\
\hline Oclusão de PDA & 56 & $\begin{array}{c}41,9 \\
(27-71)\end{array}$ & 443 & $\begin{array}{c}37 \\
(72-217)\end{array}$ & 266 & $\begin{array}{c}18 \\
(4-251)\end{array}$ & 467 & $\begin{array}{c}42 \\
(71)\end{array}$ & 165 & $\begin{array}{l}34,5 \\
(37)\end{array}$ \\
\hline $\begin{array}{l}\text { Fechamento do } \\
\text { dispositivo DSA/FOP }\end{array}$ & 52 & $\begin{array}{c}25,5 \\
(13-36)\end{array}$ & 295 & $\begin{array}{c}34 \\
(64-199)\end{array}$ & 345 & $\begin{array}{c}21 \\
(2-367)\end{array}$ & 568 & $\begin{array}{c}41 \\
(71)\end{array}$ & 259 / 21 & $41,9(50) / 23(30)$ \\
\hline $\begin{array}{l}\text { Fechamento do } \\
\text { dispositivo DSV }\end{array}$ & 6 & $\begin{array}{c}169,2 \\
(71-513)\end{array}$ & - & - & - & - & - & - & 32 & $\begin{array}{c}130 \\
(175)\end{array}$ \\
\hline $\begin{array}{l}\text { Angioplastia ou stent } \\
\text { VSVD/AP }\end{array}$ & 35 & $\begin{array}{c}155,9 \\
(76-224)\end{array}$ & - & - & 366 & $\begin{array}{c}102 \\
(8-910)\end{array}$ & 427 & $\begin{array}{c}132 \\
(222)\end{array}$ & - & - \\
\hline Angioplastia aórtica & 32 & 98,2 & 288 & 90 & 120 & $\begin{array}{c}43 \\
(7-447)\end{array}$ & 182 & $\begin{array}{c}66 \\
(107)\end{array}$ & - & - \\
\hline Stent aórtico & & $(42-206)$ & & & 52 & $\begin{array}{c}80 \\
(13-448)\end{array}$ & 112 & $\begin{array}{c}90 \\
(159)\end{array}$ & - & - \\
\hline Stent PDA & 6 & $\begin{array}{c}77,2 \\
(58-126)\end{array}$ & - & - & - & - & - & - & - & - \\
\hline $\begin{array}{l}\text { Implante de válvula } \\
\text { Melody }\end{array}$ & 3 & 273,8 & 199 & $\begin{array}{c}257 \\
(400-671)\end{array}$ & 38 & $\begin{array}{c}191 \\
(60-935)\end{array}$ & 88 & $\begin{array}{c}186 \\
(299)\end{array}$ & - & - \\
\hline
\end{tabular}

DAP/peso: DAP indexado por peso corporal.

Valores de DAP descritos em medianas e faixas interquartis: a (percentil 25, 75); ${ }^{\text {bi }}$ (percentil 75,95$)$;

c(Percentil 75). A angioplastia aórtica e o stent são agrupados em CHAIN e C3PO. 75th percentile).

$n=$ número absoluto de pacientes. DAP: produto dose-área; PDA: ducto arterioso patente; DAS: defeitos do septo atrial; FO: forame oval; DSV: defeito do septo ventricular; VSVD: via de saída do ventrículo direito; AP: artéria pulmonar; CHAIN: Brasilian registry of Congenital HeArt disease INtervention and angiography; C3PO: Congenital Cardiac Catheterization Project on Outcomes; CCISC: Congenital Cardiovascular Interventional Study Consortium.

\section{Referências}

1. Feltes TF, Bacha E, Beekman RH, Cheatham JP, Feinstein JA, Gomes AS, et al. Indications for cardiac catheterization and intervention in pediatric cardiac disease: a scientific statement from the American Heart Association. Circulation. 2011;123(22):2607-52.

2. Chida K, Ohno T, Kakizaki S, Takegawa M, Yuuki H, Nakada M, et al. Radiation dose to the pediatric cardiac catheterization and intervention patient. Am J Roentgenol. 2010;195(5):1175-9.

3. Smith BG, Tibby SM, Qureshi SA, Rosenthal E, Krasemann T. Quantification of temporal, procedural, and hardware-related factors influencing radiation exposure during pediatric cardiac catheterization. Catheter Cardiovasc Interv. 2012;80(6):931-6.

4. Ghelani SJ, Glatz AC, David S, Leahy R, Hirsch R, Armsby LB, et al. Radiation dose benchmarks during cardiac catheterization for congenital heart disease in the United States. JACC Cardiovasc Interv. 2014;7(9):1060-9.

5. Strauss KJ, Kaste SC. ALARA in pediatric interventional and fluoroscopic imaging: striving to keep radiation doses as low as possible during fluoroscopy of pediatric patients - a white paper executive summary. J Am Coll Radiol. 2006;3(9):686-8.
6. Chambers CE, Fetterly KA, Holzer R, Lin PJ, Blankenship JC, Balter S, et al. Radiation safety program for the cardiac catheterization laboratory. Catheter Cardiovasc Interv. 2011;77(4):546-56.

7. Verghese GR, McElhinney DB, Strauss KJ, Bergersen L. Characterization of radiation exposure and effect of a radiation monitoring policy in a large volume pediatric cardiac catheterization lab. Catheter CardiovascInterv. 2012;79(2):294-301.

8. Kobayashi D, Meadows J, Forbes TJ, Moore P, Javois AJ, Pedra CA, et al. Standardizing radiation dose reporting in the pediatric cardiac catheterization laboratory-A multicenter study by the CCISC (Congenital Cardiovascular Interventional Study Consortium). Catheter Cardiovasclnterv. 2014;84(5):785-93.

9. Dragusin O, GewilligM, DesmetW, Smans K, Struelens L, Bosmans H. Radiation dose survey in a paediatric cardiac catheterisation laboratory equipped with flat-panel detectors. Radiat Prot Dosimetry. 2008;129(1-3):91-5.

10. Al-Haj AN, Lobriguito AM, Rafeh W. Variation in radiation doses in paediatric cardiac catheterisation procedures. Radiat Prot Dosimetry. 2008;129(13):173-8. 
11. Borik S, Devadas S, Mroczek D, Lee KJ, Chaturvedi R, Benson LN. Achievable radiation reduction during pediatric cardiac catheterization: How low can we go? Catheter Cardiovasc Interv. 2015;86(5):841-8.

12. Cevallos PC, Armstrong AK, Glatz AC, Goldstein BH, Gudausky TM, Leahy RA, et al. Radiation Dose Benchmarks in Pediatric Cardiac Catheterization: A Prospective Multi-Center C3PO-QI Study. Catheter Cardiovasc Interv. 2017;90(2):269-80.
13. Ubeda C, Vano E, Miranda P, Leyton F. Pilot program on patient dosimetry in pediatric interventional cardiology in Chile. Med Phys. 2012;39(5):2424-30.

14. Onnasch DG, Schröder FK, Fischer G, Kramer HH. Diagnostic reference levels and effective dose in paediatric cardiac catheterization. Br J Radiol. 2007;80(951):177-85.

15. Glatz AC, Patel A, Zhu X, Dori Y, Hanna BD, Gillespie MJ, et al. Patient radiation exposure in a modern, large-volume, pediatric cardiac catheterization laboratory. Pediatr Cardiol. 2014;35(5):870-8. 